\title{
La evaluación de la Resolución de Problemas de Matemáticas de profesores de Secundaria en Colombia
}

\section{The assessment of mathematical problem solving by secondary teachers in Colombia}

\author{
Janeth A. Cárdenas Lizarazo ${ }^{1}$ \\ jacardenas/@unex.es \\ Lorenzo J. Blanco Nieto \\ Iblanco@unex.es \\ Universidad de Extremadura, España
}

\begin{abstract}
Resumen:
Presentamos los resultados de una investigación sobre la evaluación de la resolución de problemas de matemáticas (RPM) que llevan a cabo los profesores de secundaria en Bogotá (Colombia). Los resultados se obtuvieron a través de la triangulación de los análisis estadísticos sobre las respuestas dadas por profesores a un cuestionario de elaboración propia y del análisis de contenido realizado sobre los instrumentos de evaluación y las intervenciones en los grupos de discusión en los que participaron algunos de estos profesores. Por lo tanto, la investigación utiliza herramientas de tipo cuantitativo y cualitativo.

La triangulación se hizo a partir de las categorías comunes o afines entre los diferentes instrumentos de investigación y nos permitió acercarnos a las prácticas de evaluación de los profesores cuando decían que evalúan la RPM. Resultado de ello concluimos que existe cierto inmovi-
\end{abstract}

\begin{abstract}
:
This study presents the results of a research on the assessment of mathematical problem solving (MPS) developed by secondary teachers in Bogotá city (Colombia). These results were obtained by means of the triangulation of the statistical analysis over the answers given by teachers to a questionnaire prepared by the author, the content analysis of the assessment instruments and the interventions in the discussion groups in which some of these teachers participated. Therefore, the research uses quantitative and qualitative tools. The triangulation was carried out by defining the common or similar categories obtained through the different research instruments and allowed us to approach teachers' evaluation practices. We concluded that there exist certain immobility and insecurity in the mathematics teachers surveyed when addressing MPS and its evaluation in the classroom. This is justified by the lack of information about
\end{abstract}

1 Dirección para correspondencia (correspondence address):

Janeth A. Cárdenas Lizarazo. Departamento de Didáctica de las Ciencias Experimentales y de las Matemáticas, Facultad de Educación, Universidad de Extremadura. Dirección postal: Avenida de Elvas s/n, CP. 06006, Badajoz (España). 
La evaluación de la Resolución de Problemas de Matemáticas de profesores de Secundaria en Colombia

Janeth A. Cárdenas Lizarazo y Lorenzo J. Blanco Nieto

lismo e inseguridad por parte del profesorado de Matemáticas al abordar la RPM y su evaluación en el aula justificada por el desconocimiento sobre este contenido y sobre otros instrumentos de evaluación que sean económicos y a la falta de materiales. Esta investigación forma parte de un proyecto de investigación más amplio (MEC18D502), del que derivó una tesis doctoral (Cárdenas, 2014).

\section{Palabras clave:}

Resolución de problemas; profesores de secundaria; evaluación; matemáticas; tareas de evaluación. this content, or other low-cost evaluation instruments, and by the lack of materials. This research is part of the research project (MEC18D502), from which a doctoral thesis was published (Cárdenas, 2014).

\section{Key words:}

Problem solving; secondary teachers; assessment; mathematics; assessment task.

\section{Résumé:}

Nous présentons les résultats d'une enquête sur l'évaluation de la résolution de problèmes de mathématiques (RPM) dont s'occupent les professeurs d'enseignement secondaire à Bogota (Colombia). Les résultats ont été obtenus grâce au recoupement des analyses statistiques des réponses données par les professeurs à travers un questionnaire développé par nos soins et de l'analyse des contenus réalisés sur les instruments d'évaluation et les interventions dans les groupes de discussion auxquels ont participé certains de ces professeurs. Par conséquent, la recherche utilise des outils quantitatifs et qualitatifs.

Le recoupement des informations a été effectué à partir de catégories communes ou contiguës parmi les instruments de recherche et cela nous a permis de nous approcher des méthodes d'évaluation des professeurs quand ils disaient évaluer la RPM. Nous avons conclu qu'il existe un certain immobilisme et une certaine insécurité de la part de professeurs de mathématiques quand ils abordent la RPM et leur évaluation dans la salle de classe justifiée par l'ignorance de ce type de contenu et des autres instruments d'évaluation qui sont plus économiques ainsi que le manque de matériel. Cette étude fait partie d'un projet de recherche d'une plus grande ampleur (MEC18D502), duquel en a découlé une thèse (Cárdenas, 2014).

\section{Mots clés:}

Résolution de problème; enseignants du secondaire; évaluation; matematiques; tâche d'évaluation.

Fecha de recepción: 9-5-2018

Fecha de aceptación: 12-10-2018

\section{Introducción}

La Resolución de Problemas de Matemáticas (RPM) forma parte de los avances científicos y tecnológicos, siendo una herramienta fundamental en la evolución del conocimiento matemático y piedra angular en la educación Matemática. Así, en la enseñanza/aprendizaje de las Matemáticas promueve contenidos matemáticos y a la vez favorece el desa- 
rrollo de conocimientos, habilidades y destrezas necesarias para realizar razonamientos en profundidad y hacer frente a situaciones problemáticas reales y cotidianas (Aksoy, Bayazit y Kirnap, 2015; Sánchez-Barbero, 2017). Esta idea justifica que la RPM aparezca en los currículos de matemáticas de diversos países y sea una referencia básica en todos ellos (Pino, 2013). Su presencia puede ser dotada como un objetivo de aprendizaje a desarrollar entre los diferentes contenidos, como un contenido propio, como una competencia transversal en el aprendizaje de las matemáticas o como metodología de enseñanza (Puig, 2008).

Santos (2012) indica que asumir la RPM como una competencia implica hacer uso de la matemática en un contexto concreto (aspectos cognitivo-conceptuales), desarrollar recursos y habilidades para comprender conceptos y aprender específicamente a resolver problemas matemáticos (aspectos cognitivo-heurísticos). A esta referencia es a lo que Cárdenas y Blanco, (2015) Ilaman Resolución de Problemas como contenido, e incluyen en ella las creencias, emociones y actitudes (Dominio Afectivo) que se pueden suscitar este proceso (aspectos afectivos). Ortega, Pecharroman y Sosa (2011) indican que la RPM han ser desarroIladas usando situaciones que sean aceptadas por los estudiantes, les generen un bloqueo al no saber cómo abordar el problema y se esfuercen por buscar nuevos métodos para afrontar el problema.

Según Lester (2013) la mayoría de los trabajos que tratan sobre la Resolución de Problemas (RP) se centran en el resolutor del problema o el tipo de tareas que se plantean, prestando menor atención al rol del profesor y a lo que ocurre en el aula de clases. Este mismo autor afirma que es necesario unificar las teorías que se han generado en torno de la RPM desde hace más de 30 años, tales como: i) reformular lo que se entiende por problema y por RP, ii) mejorar las habilidades metacognitivas del alumnado, iii) que el profesor sea competente resolviendo problemas y no un simple experto solucionador de problemas, iv) reconocer la RP como una metodología de investigación y v) considerar la RP como una actividad cognitiva de alto nivel.

La evaluación del aprendizaje se considera como un elemento fundamental del proceso de enseñanza-aprendizaje donde señalamos dos consideraciones generales: una función pedagógica para profundizar en los aprendizajes de los estudiantes y de certificación donde el profesor califica los aprendizajes adquiridos por los estudiantes. Conviene recordar que la práctica evaluativa dota de importancia los contenidos que 
son evaluados, ya que todo lo que es objeto de evaluación se convierte en objeto de estudio para los estudiantes (Harlen, 2012), Ilegando a ignorar todo aquello que no es evaluado (Vallejo y Molina, 2014). Esto mismo es aplicable a la RP como contenido, específico o trasversal.

Los instrumentos que existen actualmente para evaluar la RPM son diversos y permiten a los profesores leer, escuchar y observar-registrar, para evaluar, lo que el alumno escribe, habla o hace (Cárdenas, 2014). Sin embargo, la evaluación se sigue haciendo mayoritariamente a través de registros escritos, usando fundamentalmente el examen escrito donde se evalúa la aplicación de las matemáticas para resolver problemas con actividades que, generalmente, requieren el uso de uno o más algoritmos, a través del lenguaje textual (Cárdenas, Blanco y Cáceres, 2016; Remesal, 2006). Este resultado evidenciaría que los profesores de matemáticas dejarían fuera diferentes aspectos de la RP ya que se utilizaría como medio para evaluar determinados contenidos y procesos matemáticos.

Recordamos que los currículos presentan indicaciones y aspectos a desarrollar sobre los contenidos a trabajar en los diferentes niveles académicos, entre ellos la RP (Pino, 2013), y el profesor es quien decide implícita o explícitamente los contenidos que trata en el aula de clase, las metodologías a seguir para su enseñanza y evaluación (Álvarez y Blanco, 2015).

Los cambios en la legislación educativa o en el currículo no implican necesariamente cambios en la práctica docente ni en evaluación que Ilevan a cabo los profesores. Álvarez y Blanco (2015) y Grupo de Investigación en Evaluación (2008) indican un cierto inmovilismo y una actuación no acorde con las propuestas de cambio sugeridas en las propuestas Curriculares y, más específicamente, sobre la evaluación. Lo que puede deberse, a las concepciones que tienen los profesores sobre la evaluación o sobre los contenidos que evalúan (Prieto y Contreras, 2008).

En este sentido, Lester (2013) indica que es de interés el profundizar sobre el conocimiento profesional que posee el profesor de matemáticas en la RP. Cárdenas, Blanco, Guerrero y Gómez (2013) indican que en la evaluación de la RP se prioriza la evaluación de lo cognitivo sobre lo afectivo y de lo algorítmico sobre lo heurístico, señalando que hay algunas contradicciones en algunos aspectos que los profesores consideran importantes a evaluar y no evalúan, llegando a evaluar cuestiones que consideran menos importantes. En Cárdenas, Blanco, Guerrero y Caballero (2016) se confirman dichos resultados a través de análisis inferenciales, y se indica que existe relación estadísticamente significativa 
entre los aspectos que los profesores manifiestan que es muy importante evaluar y los que indican evaluar, pero también se muestran aspectos sobre los cuales no existe dicha relación. Al encontrarse este resultado y encontrar diferencias estadísticas entre lo que se manifiesta que se hace y lo que se piensa que debería ser, se hace necesario determinar si esto se debe a la reflexión personal, a la deseabilidad social o al azar.

Además, en el currículo no solo se describen aspectos cognitivo-conceptuales, cognitivo-heurísticos y afectivos que intervienen en la RPM, también se sugieren otra serie de cuestiones que diferentes autores han tratado de caracterizar al analizar las actividades que se les proponen a los estudiantes en la RPM. A este respecto, se hace referencia al tipo de contenido evaluado, el soporte comunicativo utilizado e implicado y la naturaleza de la tarea atendiendo al contexto, definición del punto de partida y demandas adicionales (Díaz y Poblete, 2001; Fortuny, 2000; Remesal, 2006; entre otros).

Nuestro objetivo general es: describir las dificultades y fortalezas que los profesores de matemáticas de secundaria encuentran en sus prácticas de la evaluación de la RPM a partir de sus manifestaciones sobre lo que piensan y hacen al evaluar la RP, y lo que evalúan a través de los instrumentos que emplean para ello.

Teniendo en cuenta estas tres variables (Resolución de problemas de Matemáticas, Evaluación y Profesorado) en este documento presentamos algunos resultados acerca de qué y cómo se evalúa la RP en secundaria en los colegios públicos de Bogotá (Colombia), obtenidos con tres de los instrumentos de investigación utilizados por Cárdenas (2014) y que describimos en el siguiente apartado.

\section{Población de estudio y metodología de investigación}

\section{Población de estudio}

La muestra de 211 profesores de matemáticas de secundaria y bachillerato en los colegios públicos y urbanos de la ciudad de Bogotá (Colombia) es aleatoria estratificada, por zona y jornada, y por disponibilidad. Su rango de edad varía entre los 24 y los 64 años, donde la edad promedio es de 42 años, y con una desviación típica de 10,19 años, aunque la moda es de 30 años. Un 54\% de ellos son hombres y un $46 \%$ son mujeres. 
Es de destacar que de estos profesores un 9,5\% tiene una experiencia laboral menor a 5 años, un 22,9\% entre los 6-10 años, un 19,4\% entre los 11-15 años, un 10,9\% entre los $16-20$ años y un $37,3 \%$ mayor a los 20 años. El tamaño de la muestra varía en los instrumentos de evaluación aplicados pero, todos los sujetos forman parte de la misma muestra.

\section{Instrumentos de investigación}

Realizamos un primer acercamiento a los profesores a través de un cuestionario sobre la evaluación de la resolución de problemas (CERP) en busca de reconocer los aspectos que afirman evaluar, o no, de la RPM, y si los consideran, o no, importante su evaluación. Participaron los 211 profesores. A todos se les pidió que nos facilitaran los instrumentos de evaluación (IE) que ellos utilizan para evaluar la RPM, al objeto de caracterizar los aspectos que evalúan en su práctica docente. A este respecto, y dado que nuestro objetivo no es estudiar las concepciones de los profesores sobre la RPM, los instrumentos de evaluación se solicitaron significándole específicamente que "necesitamos que nos faciliten los instrumentos que emplean para evaluar la RP", 120 profesores nos entregaron algún IE. Finalmente, se realizaron 12 Grupos de Discusión (Gil, 1992-1993), con 37 profesores previamente seleccionados, a partir del análisis especulativo realizado sobre los resultados obtenidos con el CERP y los IE (Woods, 1987), en los cuales se comentaron los resultados encontrados y ellos pusieron de manifiesto diferentes dificultades que sienten para evaluar dicho contenido.

- Cuestionario sobre la evaluación de la resolución de problemas $(C E R P)$. Es de elaboración propia e indaga fundamentalmente aspectos que el currículo de matemáticas de Colombia señala sobre la RP (Cárdenas, Blanco, Guerrero y Gómez, 2013). Consta de tres bloques (B1: aspectos que se evalúan; B2: aspectos que se tienen en cuenta para planificar la evaluación; B3: aspectos sobre la enseñanza de la RP) los dos primeros bloques contienen una doble pregunta por cada ítem, se indaga si evalúa/tiene en cuenta) al evaluar la RP y el nivel de importancia que le otorgan (independientemente de si lo evalúan/tiene en cuenta). Esto se hace a partir de una pregunta dicotómica y una pregunta en escala tipo Lickert de 0 a 4 en cada ítem (Imagen 1). El bloque tercero solo indaga por el nivel de acuerdo en una escala Lickert de 1 a 4. 
1. Para evaluar si sus alumnos comprenden el problema ¿Evalúo?

\begin{tabular}{|l|l|l|}
\hline Si & No & $\begin{array}{l}\text { El distinguir y separar por partes un proble- } \\
\text { ma hasta comprenderlo }\end{array}$ \\
\hline $\mathrm{Si}$ & No & $\begin{array}{l}\text { El explicar lo que personalemente se conci- } \\
\text { be o entiende de un problema }\end{array}$ \\
\hline $\mathrm{Si}$ & No & $\begin{array}{l}\text { El visualizar las relaciones existentes entre } \\
\text { los diferentes elementos del problema. }\end{array}$ \\
\hline $\mathrm{Si}$ & No & $\begin{array}{l}\text { El hacer pronósticos sobre la solución del } \\
\text { problema dado }\end{array}$ \\
\hline
\end{tabular}

\begin{tabular}{|c|c|c|c|c|}
\hline \multicolumn{5}{|c|}{$\begin{array}{c}\text { Nivel de } \\
\text { importancia }\end{array}$} \\
\hline Nada & $\mathrm{Pc}$ & & Mu & \\
\hline 0 & 1 & 2 & 3 & 4 \\
\hline 0 & 1 & 2 & 3 & 4 \\
\hline 0 & 1 & 2 & 3 & 4 \\
\hline 0 & 1 & 2 & 3 & 4 \\
\hline
\end{tabular}

Imagen 1. Ejemplo de 3 ítems del CERP y su doble pregunta.

Sobre las respuestas obtenidas a este cuestionario se hacen diversos análisis estadísticos haciendo uso del SPSS 20: descriptivo, inferencial y multifactorial, con el fin de caracterizar lo que se afirma (o no) evaluar, a lo que se le da mayor (o menor) importancia en la evaluación (Cárdenas, Blanco, Guerrero y Gómez, 2013), el nivel de asociación entre el afirmar que se evalúa y el nivel de importancia que se otorga (Cárdenas, Blanco, Guerrero y Caballero, 2016), y el realizar grupos poblacionales de docentes que dan mayor importancia a ciertos aspectos respecto a otros (Cárdenas, 2014). El análisis de los resultados se organiza por categoría de análisis (Cuadros 1 y 2) establecidas a partir de la literatura sobre resolución de problemas ya citada.

Cuadro 1. Aspectos que se pueden evaluar o tener en cuenta en la planificación de la evaluación de la resolución de problemas.

\section{Aspectos evaluables}

\begin{tabular}{|c|c|c|c|}
\hline & Bloque 1 & Bloque 2 & Bloque 3 \\
\hline $\begin{array}{l}\text { Cognitivo } \\
\text { conceptual }\end{array}$ & $13,14,20$ & $49,50,54$ & $59,61,71,81$ \\
\hline $\begin{array}{l}\text { Cognitivo } \\
\text { heurístico }\end{array}$ & $\begin{array}{c}1,2,3,4,5,6,7,8,9,10,11, \\
12,15,16,17,18,19,29\end{array}$ & $\begin{array}{c}37,38,41,48, \\
55,56\end{array}$ & $\begin{array}{c}60,62,72,74, \\
75\end{array}$ \\
\hline Afectivo & $21,22,23,24,25,26,27,28$ & $\begin{array}{c}39,40,51,52, \\
53,57\end{array}$ & 65,73 \\
\hline
\end{tabular}


La evaluación de la Resolución de Problemas de Matemáticas de profesores de Secundaria en Colombia

Janeth A. Cárdenas Lizarazo y Lorenzo J. Blanco Nieto

Cuadro 2. Aspectos sobre los que se enfatiza al planificar en la evaluación de la resolución de problemas.

\begin{tabular}{|c|c|c|c|}
\hline \multicolumn{4}{|c|}{ Características de los problemas } \\
\hline & Bloque 1 & Bloque 2 & Bloque 3 \\
\hline Contextos & & $30,31,42,43$ & 77 \\
\hline Tipo/Número de respuestas & 18 & $32,33,34,35,36$ & 58 \\
\hline Datos/Enunciado & & & $\begin{array}{l}76,78,79,80, \\
82,83,84,85 \\
\end{array}$ \\
\hline Tipo de actividades & & $44,45,46,47$ & \\
\hline $\begin{array}{l}\text { Lo que se espera de los } \\
\text { estudiantes }\end{array}$ & & & $\begin{array}{c}63,64,66,67, \\
68,69,70\end{array}$ \\
\hline
\end{tabular}

- Instrumentos de evaluación con los que se evalúa la Resolución de Problemas (IE). De los 211 profesores, 120 profesores facilitaron los instrumentos de evaluación, obteniéndose en total 84 instrumentos. El estudio de procedencia de estos profesores mostró que había de todas las zonas consideradas y de las jornadas de trabajo. Se identificaron los enunciados-actividades y se analizan las diferentes tareas que aparecían en estos (Imagen 2). Cada tarea se analizó a partir de las categorías de análisis previamente establecidas (Cuadro 3) con el fin de caracterizar las prácticas para evaluar.
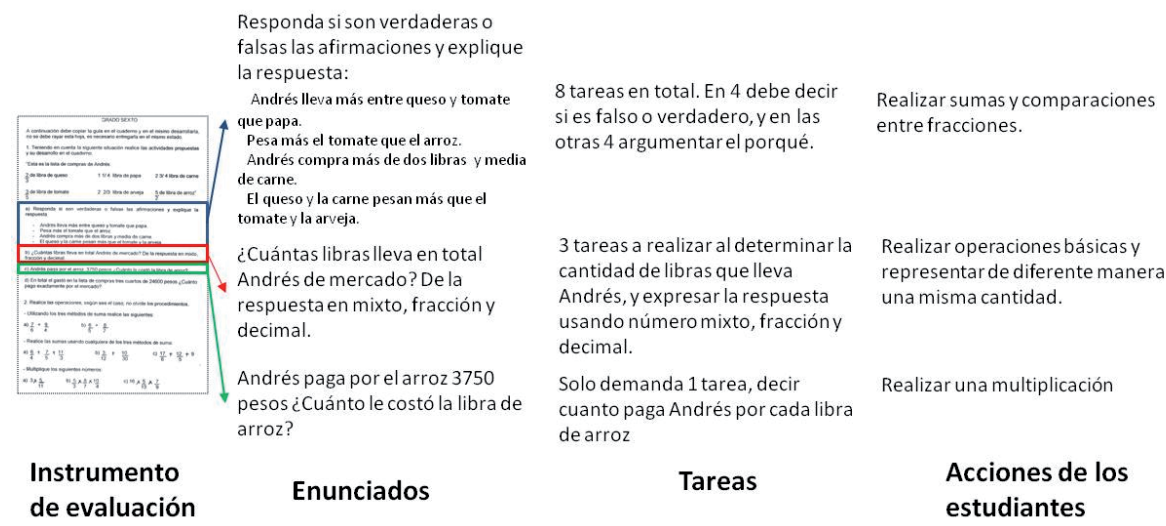

Tareas

Acciones de los estudiantes

Imagen 2. Esquema seguido para el análisis de las tareas que figuran en cada instrumento de evaluación. 
Cuadro 3. Categorías y subcategorías para el análisis de las tareas de evaluación (Tomado de Cárdenas, Blanco y Cáceres, 2016, p. 66).

\begin{tabular}{|c|c|c|}
\hline \multirow{11}{*}{$\begin{array}{l}\text { Tipos de conteni- } \\
\text { dos evaluados/Exi- } \\
\text { gencia cognitiva } \\
\text { en la tarea }\end{array}$} & Conceptuales & $\begin{array}{l}\text { Nivel } 1 \text {. Recuerdo directo de informa- } \\
\text { ción factual. }\end{array}$ \\
\hline & & $\begin{array}{l}\text { Nivel } 1 \text {. Identificación y ejemplifica- } \\
\text { ción entre conceptos. }\end{array}$ \\
\hline & & $\begin{array}{l}\text { Nivel 2. Establecimiento de relaciones } \\
\text { entre conceptos. }\end{array}$ \\
\hline & & $\begin{array}{l}\text { Nivel 3. Explicación/modelización de } \\
\text { un fenómeno complejo mediante el } \\
\text { uso integrado de una red de conceptos } \\
\text { interrelacionados. }\end{array}$ \\
\hline & Procedimentales & $\begin{array}{l}\text { Nivel 1. Aplicación directa de algorit- } \\
\text { mos o técnicas. }\end{array}$ \\
\hline & & $\begin{array}{l}\text { Nivel } 1 \text {. Inerpretación/Traducción en- } \\
\text { tre lenguajes o formas de representa- } \\
\text { ción/conversión. }\end{array}$ \\
\hline & & $\begin{array}{l}\text { Nivel 2. Identificación de algorino a } \\
\text { aplicar y aplicación del mismo. }\end{array}$ \\
\hline & & $\begin{array}{l}\text { Nivel 2. Identificación y aplicación en- } \\
\text { cadenada de los algorítmos a aplicar. }\end{array}$ \\
\hline & & $\begin{array}{l}\text { Nivel 3. Delimietación y concreción } \\
\text { de la formulación del problema y/o } \\
\text { utilización de estrategias o heurísticos } \\
\text { para su resolución. }\end{array}$ \\
\hline & Metacognitivos & $\begin{array}{l}\text { Nivel 1. Verificación de capacidades, } \\
\text { habilidades, dificultades,... }\end{array}$ \\
\hline & & $\begin{array}{l}\text { Nivel 2. Autovaloración de capacida- } \\
\text { des, habilidades, dificultades,... }\end{array}$ \\
\hline
\end{tabular}

6. Soporte comuni- Verbal cativo utilizado. Numérico

7. Soporte comuni- Tabular cativo implicado Imagen

\begin{tabular}{|c|c|c|c|}
\hline & \multicolumn{3}{|c|}{ Gráfico } \\
\hline & \multicolumn{3}{|c|}{ Recurso manipulativo } \\
\hline \multirow{6}{*}{$\begin{array}{l}\text { 8. Naturaleza de la } \\
\text { tarea }\end{array}$} & \multirow{6}{*}{$\begin{array}{l}\text { Contexto en que } \\
\text { se inscribe la tarea }\end{array}$} & \multicolumn{2}{|l|}{ Real } \\
\hline & & \multicolumn{2}{|c|}{ Realistico } \\
\hline & & \multirow[t]{2}{*}{ Ficticio } & Situación no real \\
\hline & & & $\begin{array}{l}\text { Matemáticamente no des- } \\
\text { cribe la situación }\end{array}$ \\
\hline & & \multicolumn{2}{|c|}{ Intramate- Manipulativo } \\
\hline & & mático & Recreativo \\
\hline
\end{tabular}




\begin{tabular}{|c|c|}
\hline \multirow{3}{*}{$\begin{array}{l}\text { Definición del } \\
\text { punto de partida } \\
\text { de la tarea. Datos } \\
\text { e informaciones } \\
\text { que se ofrecen }\end{array}$} & Datos necesarios y suficientes \\
\hline & Datos irrelevantes o redundantes \\
\hline & Datos insuficientes \\
\hline \multirow[t]{4}{*}{$\begin{array}{l}\text { Demandas adi- } \\
\text { cionales }\end{array}$} & $\begin{array}{l}\text { Emplear más de un proceso en la reso- } \\
\text { lución de la tarea }\end{array}$ \\
\hline & $\begin{array}{l}\text { Obtener más de una solución/produc- } \\
\text { to como resultado de la tarea }\end{array}$ \\
\hline & Justificar la tarea \\
\hline & $\begin{array}{l}\text { Inventar, formular, reformular o trans- } \\
\text { formar un problema }\end{array}$ \\
\hline
\end{tabular}

- Los Grupos de discusión (GD) permitieron debatir con los profesores los resultados obtenidos en busca de estimular su recuerdo y profundizar sobre las apreciaciones hechas e identificar las facilidades o dificultades que se plantean al momento de evaluar la resolución de problemas. Un primer análisis especulativo (Woods, 1987) nos permite describir y categorizar la información y elegir profesores y grupos de profesores representativos de los resultados obtenidos, buscando, además, que fueran buenos informantes para ayudarnos a profundizar en la información obtenida (Gil, 1992-1993). Participaron 37 profesores, de diferentes zonas y jornadas de trabajo, en alguno de los 12 GD. El Cuadro 4 presenta el guion empleado como pauta para el debate.

Cuadro 4. Guion para los grupos de discusión.

1. ¿Por qué considera usted que se le otorga un mayor nivel de importancia a evaluar aspectos que están ligados al dominio cognitivo sobre los del dominio afectivo?

2. ¿Por qué considera usted que se le otorga un mayor nivel de importancia a evaluar aspectos que están ligados a contenidos matemáticos sobre aspectos que refieren a los heurísticos?

3. Desde su experiencia ipor qué cree que hay profesores que manifiestan no evaluar aspectos y que dicen que son aspectos importantes a evaluar?

4. ¿Considera que es importante evaluar? ¿por qué?

5. ¿Considera que es importante evaluar la resolución de problemas? ¿por qué?

6. ¿Qué dificultades tiene usted para evaluar la resolución de problemas?

7. ¿Considera que es difícil evaluar los aspectos del dominio afectivo o cognitivo de la resolución de problemas? ¿Qué heurísticos considera difíciles de evaluar? ¿Qué aspectos del dominio afectivo considera difíciles de evaluar? ¿por qué? 
8. ¿Considera que la evaluación le sirve al estudiante para algo? ¿para qué? ¿cómo se puede asegurar esto?

9. Escriba con sus palabras lo que es para usted un problema. Luego responda: cuando está evaluando a sus estudiantes y plantea un problema ¿con qué finalidad lo hace? ¿Cuál considera que es el papel de la resolución de problemas en la evaluación en matemáticas?

10. ¿Considera qué hay mucha diferencia entre lo que "se manifiesta" y lo que "se hace" en las aulas?

Las discusiones duraban poco más de una hora, se grabaron y transcribieron para realizar un análisis de contenido, ubicando cada unidad de análisis en alguna(s) de la(s) 11 categorías de análisis con sus respectivas subcategorías (Cuadro 5), para ello se hizo uso del programa $\mathrm{N}$ VIVO. Las afirmaciones y respuestas dadas por alguno de los profesores en los GD eran avaladas o complementadas, generalmente, por el resto de sus compañeros y no fueron exclusivas de un GD, ya que eran ideas que se repetían en otros GD.

Cuadro 5. Algunas categorías de análisis obtenidas de las Discusiones de Grupo.

C2 La evaluación cómo... EC1 Un proceso más en la educación. (EC) EC2 Un proceso pedagógico.

C5. La enseñanza de la ERP1. La enseñanza de contenidos conceptuales resolución de problemas y la resolución de problemas.

(ERP) ERP2. La enseñanza de elementos propios de la resolución de problemas.

ERP3 Aspectos afectivos en la enseñanza de la resolución de problemas.

C6 ¿Qué se evalúa? (QE) QE1. Lo cognitivo/conceptual.

QE2. Lo conceptual/heurístico.

QE3. Lo afectivo.

QE4. Competencias.

QE5. Lo metacognitivo.

C8 Lo que determina o DD1. Estudiantes.

dificulta la evaluación. DD2. El tiempo.

(DD)

DD3. Los profesores: conocimientos y prácticas sobre la evaluación en general y de la resolución de problemas en particular.

DD4 Características de la evaluación.

DD5. Situaciones sociales.

DD6. El uso TIC/TAC. 


\section{Resultados y discusión}

Triangular la información y resultados a partir de los GD permite su ampliación y profundización al explicar, complementar o contradecir las evidencias que refieren a una misma categoría de análisis entre los diferentes instrumentos (Cuadro 6). Para presentar los resultados obtenidos con los instrumentos de investigación se hace uso de la siguiente codificación:

- GD: se hace alusión a las afirmaciones de algún profesor (0000AX00) o a alguna de los resultados encontrados por categoría (p. e. ERP1). Es de destacar que por cuestiones de espacio se pone solamente una afirmación a modo de ejemplo, ya que esta misma idea es reiterada en otros GD o apoyada en el GD que se ha presentado.

- CERP: se presentan los resultados por ítem (CERP: ítem-\%), grupo de ítems ( $\%<$ CERP: ítem $\mathrm{x}, \mathrm{x}, \ldots, \mathrm{x}<\%$ ) o categorías (p.e. CERP-DA). Dado que en este cuestionario por cada ítem hay una doble pregunta, cuando se agrega a esta notación NI/A, se hace referencia al nivel de importancia que se debe dar a evaluar ese aspecto; en caso contrario lo que se indica es si el profesorado manifiesta evaluar o no dicho aspecto.

- IE: se emplean las categorías de análisis (IE, XXX).

Cuadro 6. Categorías de análisis afines o comunes entre el CERP, IE y GD, consideradas en este trabajo.

\begin{tabular}{|c|c|c|}
\hline CERP & IE & GD \\
\hline Cognitivo conceptual & $\begin{array}{l}\text { Tipos de contenido: conceptual (Nivel } 1 \text { y } \\
\text { 2) y procedimental (Nivel } 1 \text { y } 2 \text { ) }\end{array}$ & ERP!, QE! \\
\hline Cognitivo heurístico & $\begin{array}{l}\text { Tipos de contenido: conceptual (Nivel 3), } \\
\text { procedimental (Nivel 3) y metacognitivo }\end{array}$ & $\begin{array}{l}\text { ERP2, QE2, } \\
\text { QE4, QE5 }\end{array}$ \\
\hline Afectivo & & ERP3, QE3 \\
\hline Contextos & Naturaleza de la tarea: contexto & \multirow{5}{*}{$\begin{array}{l}\text { EC, ERP, } \\
\text { QE, DD }\end{array}$} \\
\hline $\begin{array}{l}\text { Tipo/Número de } \\
\text { respuestas }\end{array}$ & $\begin{array}{l}\text { Naturaleza de la tarea: demandas adicio- } \\
\text { nales }\end{array}$ & \\
\hline Datos/Enunciado & $\begin{array}{l}\text { Naturaleza de la tarea: definición del } \\
\text { punto de partida }\end{array}$ & \\
\hline Tipo de actividades & $\begin{array}{l}\text { Naturaleza de la tarea: demandas adicio- } \\
\text { nales }\end{array}$ & \\
\hline $\begin{array}{l}\text { Lo que se espera de } \\
\text { los estudiantes }\end{array}$ & & \\
\hline
\end{tabular}


Presentamos dos resultados considerados de interés para la estructuración de los programas de formación inicial y continua del profesorado de matemáticas acerca de la caracterización de su evaluación y de los instrumentos que emplean frecuentemente, que aparecen reflejados en los instrumentos utilizados. La nomenclatura descrita permite mostrar evidencias de las cuales se extraen indicios y resultados que se mencionan en el discurso.

1. La evaluación de la RPM es asumida como la aplicación de un algoritmo o concepto. En ella no se considera la evaluación sistemática de habilidades, heurísticos o contenidos propios de la $R P$, aunque la evaluación de estos aspectos se considera importante y necesario realizarla.

- Evaluar lo cognitivo conceptual (CC). La mayoría de los profesores manifiestan evaluar la complejidad de los conceptos y la aplicación de los algoritmos, además de la realización de cálculos para la obtención de la respuesta correcta [(QE1), 89.4\%<(CERP: $13,14,20)<95.5 \%]$, e indican que casi todas las tareas están asociadas por lo menos a un contenido del currículo (IE-Contenido $94.2 \%$ ). Consideran muy importante estos aspectos en la evaluación de la RP $[87.4 \%<(\mathrm{CERP}-\mathrm{NI} / \mathrm{A}: 13,14,20)<92.4 \%$, y se justifica en que la mayoría de los profesores consideran muy importante que los estudiantes al resolver problemas utilicen el contenido que se acaba de explicar (CERP-NI/A, 59-84.9\%). Esto es coherente con la idea de la RP como aplicación de un contenido previamente explicado (ERP-1012AM05), lo que dota de utilidad a las matemáticas escolares (ERP1b-1937AT02).

ERP-1012AM05 "Realmente los temas para mí siempre han sido la clave, si sabe el tema ha resuelto el problema de manera excelente, asi ha sido siempre".

ERP1b-1937AT02 "Dicen factorización, y no encuentran una utilidad inmediata, y si se aplica un problema, le ven sentido. Pienso que no puede haber matemática sin resolución de problemas, porque es la razón de ser, si no, no valdría la pena".

Desde esta perspectiva, la enseñanza de las matemáticas tiene como objetivo su utilización en un contexto concreto, y no requiere necesa- 
riamente de la adquisición de estrategias, conocimientos o habilidades propios de la RP (Puig, 1996; Schoenfeld, 1985), por lo que su evaluación tampoco lo contiene. Puig (1996) menciona que en la enseñanza de la RP se debe ahondar en el uso de estructuras cognitivas de nivel superior, sin necesidad de ahondar en conceptos matemáticos. Por su parte, el currículo colombiano (MEN, 2007) establece la importancia de abarcar de manera complementaria y equilibrada el trabajo sobre el contenido convencional y las habilidades de pensamiento sin necesidad de hacer un mayor énfasis en alguno de esos contenidos (Nikerson, Perkins y Smith, 1994), e indican la importancia de su evaluación.

- Evaluar lo cognitivo heurístico (CH). El porcentaje de profesores que manifiestan evaluar los diferentes heurísticos o capacidades propias de la RP es mucho menor en casi todos los aspectos $[54.03 \%<(\mathrm{CERP}-\mathrm{CH})<84.83 \%$ ] respecto a la evaluación de lo cognitivo conceptual.

La poca evidencia de la evaluación de los aspectos cognitivo-heurísticos se constata en las pruebas escritas, donde la evaluación de la RP engloba el uso de recuerdo de información factual, el uso de diferentes lenguajes matemáticos y la aplicación de uno o varios algoritmos para llegar a resolver las tareas propuestas en general y aquellas que se consideran problema [IE conceptual (nivel $1=80 \%$, nivel $2=16 \%$ ), procedimental (nivel $1=55 \%$, nivel $2=41 \%$ )].

El porcentaje de profesores que manifiesta evaluar los aspectos cognitivo-heurísticos es menor que el porcentaje de profesores que pone de manifiesto el considerar que su evaluación sea importante [71.7\%<(CERP$\mathrm{NI} / \mathrm{A}-\mathrm{CH})<92.1 \%$ ]. Esto indica que hay un reconocimiento por parte de los profesores de cuestiones que consideran importantes y no evalúan o lo hacen en pequeña medida, que se reafirma en los GD. "A uno le queda en la cabeza que lo que hay que hacer es que el chino (estudiante) sea capaz de identificar variables, de modelarlas, de operarlas, de concluirlas, de..., de pasar de ese lenguaje matemático a ese..., transformarlo, pasarlo por aquí..., pronosticar, hacer eso" (QE2-1937AT02).

También, el porcentaje que manifiesta evaluar los aspectos cognitivoheurísticos es bastante menor que el de los profesores que manifiestan indicar que es muy importante tenerlos en cuenta $[84.2 \%<(\mathrm{CERP}-\mathrm{NI} / \mathrm{A}-$ CC) $<95.6 \%$. En este mismo sentido es posible afirmar que es mayor el porcentaje de docentes que manifiesta no evaluar los aspectos cognitivo- 
heurísticos $[8 \%<(\mathrm{CERP}-\mathrm{CH})<40.3 \%]$ respecto de los aspectos cognitivoconceptuales.

Esta situación nos recuerda la aportación de Santos (2007) al sugerir la necesidad de proponer tareas que capturen la información de los diferentes momentos identificados en el modelo de la resolución de problemas, indicando la necesidad de formar a los profesores de matemáticas entorno a este contenido.

- Dificultades ante la evaluación de lo cognitivo-heurístico. A pesar de no evaluar estos aspectos afirman que consideran importante tenerlos en cuenta en la evaluación $[7 \%<(\mathrm{CERP}-\mathrm{CH})<23 \%]$. Así la asociación que se puede establecer entre las respuestas que dan los profesores si evalúan y el nivel de importancia que dan a que los estudiantes expliquen lo que comprenden del problema, el establecer relaciones entre los datos o el crear otro método diferente al trabajado para RP no presentan relación estadística significativa por lo que sus respuestas se podían deber a una cuestión del azar (Prueba chi-cuadrado CERP: 2- $\mathrm{p}=.393,3-\mathrm{p}=.055,15-\mathrm{p}=.208$,). En los GD se pone de manifiesto la importancia de evaluar estos aspectos propios de la RP aunque en realidad no se evalúen. Esto lo justifican en atención a los procesos de formación de profesores y falta de recursos, en relación a la RPM y la evaluación.

DD3c-1917AT11 "...si a mí no me formaron para solucionar problemas. Por más que yo lo quiera hacer me va a ser difícil formar a mis alumnos en resolución de problemas".

DD3c-0718AM01 "...la evaluación sigue siendo exactamente igual. Es lo mismo y de la misma manera. Nos cuesta mucho hacer las pruebas de comprensión,... Entonces nosotros seguimos pegados a la tradición, a lo que siempre se hizo."

DD4c-1917AM09 "Puedo implementar plataformas educativas, puedo llevar o tener una sala de sistemas, puedo hacer laboratorios con un software matemático. Acá, eso es imposible... No tengo la oportunidad de que ellos puedan interactuar con el software, ni el acceder a un equipo".

DDC4a-0718AM01 "Generalmente lo que se hace es calificar. Es mucho más fácil decir si un muchacho hace bien un cálculo o aplica bien una ecuación... a llegar a decir, oiga explíqueme que entiende en este enunciado y qué información hay, cómo puede 
usted construir un tipo de relación ahí,..., entonces cosas como esas no requieren respuestas cerradas, se vuelven respuestas un poco más amplias, abiertas, lo que hace que usted necesite más tiempo para trabajar con ellas".

\section{Los heurísticos que manifiestan evaluar con mayor frecuencia son} comprender, modelar y comprobar la respuesta a la luz del problema $[86.9 \%<$ (CERP: 1, 7, 19)<88.8\%]. También, verbalizar lo que se entiende del problema y los procedimientos seguidos; establecer relaciones y el uso de lenguajes matemáticos y el argumentar [79.3\%<(CERP: 2, $3,5,6,10,29)<84.6 \%$ ]. Sin embargo, son escasos y, en algunos casos, cuestionables los ejemplos de las tareas que evalúan estas cuestiones (IE).

La necesidad de evaluar la comprensión, es aceptada y dotada de importancia por los profesores en los GD. Para ellos, comprender el problema es la capacidad de identificar lo que se pregunta y entender lo que hay que hacer (QE2), pero solo piden recordar o resaltar la información que está totalmente explicita en el enunciado. Esta evaluación se ve limitada por evaluar el procedimiento y la solución, y la comprensión queda relegada.

Cuando los estudiantes dicen no entender el problema, los docentes manifiestan que basta con pedirles que lean y relean el texto hasta que entiendan, sin señalar otro tipo de sugerencia más allá de la lectura (ERP2b-1028AM01). Algunos docentes manifiestan el recurrir a heurísticos, como el hacer un dibujo (ERP2b-0605AM01) y poco más.

ERP2b-1028AM01 "profe es que no entiendo acá", y yo "listo, hágame el favor y lee", y "no, profe", "entonces hágame el favor y vuelve y lee" y lo voy llevando, lo voy llevando, hasta que "a claro... profe".

ERP2b-0605AM01 "Entonces, empezamos a abordar el primer problema, y les dije...como nadie entiende nada vamos con un método: primero usted trate de interpretar el problema a través de un dibujito o lo que sea, luego saque unos datos, los datos que le da el problema ¿sí? cuando ya tenga esos datos mire qué conceptos son los que necesita".

No hemos encontrado evidencias que mostraran un conocimiento de los profesores acerca de los pasos necesarios para garantizar una correcta comprensión del enunciado en la línea de lo aportado por Lochhead 
y Mestre (1988), al distinguir entre comprensión cualitativa, cuantitativa y conceptual.

Los profesores esperan que sus estudiantes sean capaces de identificar los datos y relaciones que se establecen entre estos, y los asocien a las situaciones en las cuales se produce el mismo tipo de relaciones entre variables, de tal modo que al solucionarlas sean capaces de seguir el mismo protocolo (ERP2d-1603AT05). Reconocen que la enseñanza de aprendizajes reproductivos limita al estudiante a realizar procedimientos de manera mecánica, sin que haya una reflexión sobre las relaciones que establece entre los datos (ERP2d-1603AT05). Sin embargo, son escasas las actividades que presentan este tipo de estructura, solo se ve en los talleres y se logra hacer que los estudiantes las establezcan a través de preguntas orientadoras (ERP2d-1603AT05). Estas tareas se caracterizan por estar vinculadas bajo varias situaciones, en las cuales se pregunta por distintas cuestiones pero trabajando un mismo concepto.

ERP2d-1603AT05 "Bajo un determinado enunciado pregunto... qué información hay, qué relación hay entre esa información, o entre esos diferentes elementos de la información, qué tipo de relación hay, cómo puede usted construir un tipo de relación ahí". ERP2d-1603AT05 "...todavía se tiene como ese rezago, de una manera muy bien cimentada, la formación tradicional. Yo explico, usted repite. Enseño, digo como hacerlo, qué hacer y usted lo repite. Pero al cambiar una a, por una $b$, un 3 por un 4, él no va a entender, porque las relaciones no están claras, no hay ese establecimiento de esa condición. El establecimiento de condiciones entre variables, entre procedimientos de una estructura, o sea, no hay claridad".

Heurísticos como proponer varias estrategias de solución, validar modelos, implementar recursos apropiados, usar estrategias diferentes a las trabajadas en clase, mirar si la respuesta es un dato exacto o aproximado y el obtener conclusiones, son, al parecer, los segundos aspectos más evaluados [72.5\%<(CERP: 8, 11, 12, 15, 18, 29)<76.7\%]. En los instrumentos de evaluación se encuentra que el emplear estrategias de solución diferentes a las trabajadas en clase se evalúa en las olimpiadas matemáticas $(2.17 \%)$, el obtener respuestas estimadas o aproximadas en algunos exámenes $(0.36 \%)$, así como el sacar conclusiones en algunos talleres. 
Los profesores ponen de manifiesto que es muy importante crear o buscar estrategias diferentes a las trabajadas y su evaluación (CERP: 1587.9\%). Así mismo, consideran poco importante que los estudiantes sigan el camino que él quiere cuando resuelven problemas (CERP: 61$82.5 \%$ ) y en menor medida el que busquen otros caminos al finalizar la solución de un problema (CERP: 62-74.4\%). En las DG los profesores expresan que los alumnos siempre esperan a que el profesor indique el procedimiento a emplear para resolver los problemas propuestos (ERP11603AT05). Así mismo, se encuentran varios indicios de que los profesores suelen limitar a los estudiantes a emplear el procedimiento que ellos desean a través de diferentes estrategias.

ERP1-1603AT05 "En el bachillerato esperan a que el profesor le de todo el historial, le diga todo, como se hace, cual es el procedimiento y cuales los pasos a seguir. Entonces es ahí cuando el profesor le dice como es la receta".

Hay dos niveles diferentes de estrategia para RP, si es intuitiva y si es matemática. Esta última tiene un mayor valor. No se valora de igual manera un procedimiento empleado para resolver el problema que sea diferente al que el profesor ha trabajado en clase (EC2d-0718AM01).

Verificar a la luz del problema la respuesta es un aspecto escasamente demandado en las tareas propuestas, estas se refieren a verificar datos exactos, donde no se hace necesario, por ejemplo, el uso de la estimación para ver lo razonable de la respuesta encontrada en el contexto del problema.

2. Los instrumentos de evaluación que se emplean para evaluar la $R P$ son limitados en su formato y estructura.

Los IE que los profesores facilitan y emplean para evaluar la RP son pruebas escritas, aun reconociendo la insuficiencia de este tipo de pruebas para evaluar la RP por diferentes circunstancias:

- La estructura de las pruebas de evaluación. La mayoría de actividades propuestas en las pruebas escritas (54\%) demandan la selección de la respuesta correcta. En ellas, los alumnos no dejan registro de sus ideas, procedimientos, o anotaciones que han seguido para llegar a la solución de la tarea propuesta, ya que lo que se valorará es si ha marcado o no la respuesta correcta (DD4c-1901AM01). El uso de este tipo de pruebas deviene de que se requiere menos tiempo para calificarlas, que cuando son 
pruebas con preguntas abiertas (DD4c-1917AT06). Estas pruebas al estar constituidas por preguntas de selección múltiple y que se deben responder en un tiempo definido, hace que las respuestas dadas por los estudiantes no necesariamente sean resultado de la reflexión de sus conocimientos, basta con que ellos señalen una de las opciones de cada ítem, para dar por solucionado el examen (DD4c-1027AT08).

Los problemas planteados se caracterizan por ser "problemas tipo", generalmente tomados del libro de texto y/o exámenes anteriores (Álvarez y Blanco, 2015). Los profesores consciente o inconscientemente se identifican con dichas tareas, visualizan los conocimientos, estrategias o habilidades que considera pertinentes a evaluar, sin tener presente que aspectos de los que evalúa, son propios de la resolución de problemas. Conviene recordar que el MEN (2007) enfatiza que el profesor debe imaginar y proponer a los alumnos situaciones que puedan vivir y en las que los conocimientos van a aparecer como la solución óptima y descubrible en los problemas planteados.

DD4c-1901AM01 "... [en el examen] solamente alcanzo a evaluar la respuesta. No puedo ver cómo él lo podría haber hecho. Pero evidentemente hay evaluaciones que si hacemos en el periodo que me permiten identificar esa parte".

- Las características de las tareas propuestas. La mayoría de tareas propuestas evalúa recordar información factual e identificar y aplicar algoritmos para su resolución, sin dar cuenta de lo procesos comunicativos o de interacción donde se registren las ideas, razonamientos, discusiones, etc., que han surgido durante la consecución de la respuesta.

- Prima el uso de contextos matemáticos frente a los no matemáticos. El tipo de contextos que más se emplean en la evaluación de la RP son los contextos matemáticos frente a los no matemáticos [(CERP 30-98\%; 31-81.5\%) e (IE- 9\% Contexto ficticio; 70\% contexto intrammatemático)]. En la revisión de los contextos empleados en aquellas tareas que los profesores consideran problema, se observa que la mayoría de contextos son realísticos, con referencias muy tradicionales, al igual que en Blanco, Guerrero y Caballero (2013).

- El lenguaje verbal y/o numérico es mayoritariamente empleado 
al plantear las tareas de evaluación y el lenguaje numérico suele ser el lenguaje requerido para dar respuesta a la tarea propuesta. El lenguaje tabular y gráfico son los que menos se emplean y a su vez los que menos se demandan. Coinciden estos resultados con los estudios de Pino y Blanco (2008) y Remesal (2006). De modo similar sucede con el uso de las imágenes los cuales se usan para dar "vistosidad". Este uso abusivo del uso del lenguaje verbal-numérico en las actividades de texto escrito va en detrimento de otras formas de representación como tablas, gráficas o imágenes, lo cual ha sido puesto de manifiesto en ocasiones anteriores (Pino y Blanco, 2008; Remesal, 2006).

- La mayoría de los profesores considera oportuno que los alumnos tengan que inventar o formular problemas (CERP: 44-72.2\%; $45-77.2 \%$ ), y reformular o transformar problemas (CERP: 46$65.3 \%$; 47-59.4\%) cuando se evalúa la resolución de problemas. Sin embargo, tan solo 1 de las 2483 tareas analizadas demanda el inventar una situación a partir de los datos dados, lo que justifican en los GD con sentimientos de incapacidad para proponer este tipo de actividades (DD3c-0605AM04). Inventar o formular problemas, tal y como señalan los Lineamientos Curriculares o el currículo en España (Cárdenas y Blanco, 2015), está fuera de la evaluación. Recordamos la aportación de Ayllón (2012) en la que señala la importancia de formular preguntas ante diferentes situaciones o inventar problemas en la evaluación de la resolución de problemas, además de indicar expresamente, que en la formación del profesor ha de estar incluido aprender a formular preguntas adecuadamente y motivar a los estudiantes para que inventen preguntas y problemas y respondan adecuadamente.

- El desconocimiento del profesorado la evaluación de la RPM. La complejidad que abarca la evaluación de la RP lleva a algunos docentes a manifestar que no se sienten capacitados para trabajar la RP (DD3c) por la falta de claridad para realizar la conexión entre problemas de contextos realísticos con las matemáticas escolares que deben trabajar (DD3c) o no saber enunciar actividades de evaluación que midan aspectos concretos de la RP, y que se puedan cuantificar (DD3b). 
Estas declaraciones justificarían el inmovilismo en las prácticas de evaluación de la RPM. Pero también puede deberse, según Mollà (1997), a la idea de que todos sabemos evaluar y que más o menos lo hacemos por igual, aunque para ello los profesores ponen en práctica la intuición y el imitar los modelos que conocieron cuando eran estudiantes (González, Arévalo y Henao, 2009; Grupo de investigación, 2008). Esto hace que el examen final siga siendo empleado como instrumento de evaluación representativo atribuyéndole un peso significativo, aun cuando se reconoce que es un instrumento reductor al no cubrir diversos aspectos de la matemática.

En los GD los profesores indican que la evaluación de la RP solo es posible a través de los procesos de verbalización (DD5c), ya que permiten visualizar los avances que tienen los resolutores, e identificar los buenos estudiantes resolviendo problemas (QE2). No obstante, la evaluación de los procesos de verbalización es escasa.

Para los profesores la evaluación a través de procedimientos de verbalización es compleja y difícil de utilizar; asumen que estudiantes tienen dificultades para explicitar, oralmente y por escrito, el procedimiento que siguen para resolver una pregunta, (DD5c-0718AM01) y que no cuenta con los instrumentos de evaluación adecuados para ello.

DD5c-0718AM01 "Ese tipo de trabajo es difícil y todo... en un examen uno no los escucha. Esa es la excusa. Tienen problemas para hablar y aún más para escribir, para justificar algunas respuestas. Hasta los que reconocen que saben del tema, pero les cuesta justificar la respuesta".

DD2a-1012AM05 "No es sencillo evaluar esas cosas que uno considera que son importantes. En el momento en que uno tiene un grupo numeroso le queda más sencillo aplicar una prueba que me va a decir si está bien o está mal, y punto. Y no evaluar eso que necesita más tiempo, sentarse uno por uno, mirar, y si... yo considero desde mi visión es más por eso. Que uno coge lo más fácil".

\section{- Los profesores asumen las pruebas escritas como la principal he- rramienta para evaluar la RP.}

Las pruebas escritas son la herramienta fundamental en la evaluación a pesar de que consideran que este tipo de pruebas no dan cuenta de lo procesos comunicativos o de interacción con los estudiantes, ni de acciones que desarrollan en diversas tareas manipulativas o de trabajo en grupo. Como consecuencia, el pro- 
fesorado no tiene en cuenta la diversidad de formas de expresión que tienen los estudiantes, de sus conocimientos, para verificar y examinar lo aprendido (Jarero, Aparicio y Sosa, 2013), aunque durante las clases, es normal que se den diferentes tipos de comunicaciones entre el profesor y el estudiante. A este respecto, no se califica y/o no se lleva un registro de las diferentes cuestiones que verbalizan los estudiantes, en el desarrollo del trabajo de clase. Es decir, se omiten la valoración de los pensamientos de los estudiantes, sus dudas, sus conjeturas e ideas o los procesos de reflexión, entre otras cuestiones, que se recomiendan evaluar en Meier (1992) y Worth (1990). Y omitiendo otros aspectos como los que trabaja Carrillo (1995).

\section{Resumen de los resultados}

1. Las actividades de evaluación de la RP evalúan la aplicación o recuerdo de un algoritmo o concepto, primando problemas de contextos realísticos muy tradicionales.

2. Las pruebas escritas son el principal instrumento para la evaluación, aunque reconocen sus limitaciones.

3. Las actividades de evaluación están sacadas, usualmente, de los libros de textos o de exámenes anteriores.

4. En general, los instrumentos de evaluación que se emplean para evaluar la RP son limitados en su formato y estructura. Y son muy tradicionales.

5. No se considera la evaluación sistemática de habilidades, heurísticos o contenidos propios de la RP, aunque la evaluación de esta se considera importante y necesario llevarla a cabo.

6. La evaluación de los aspectos cognitivos-heurísticos es muy escasa, que justifican por las dificultades de su evaluación y falta de conocimiento, capacidad y herramientas para ello.

\section{Conclusiones}

En general, la evaluación de la RP se equipara con el recuerdo y reproducción de procesos matemáticos (usualmente, algebraicos o aplicacio- 
nes de fórmulas) en los que no se demanda otros contenidos específicos de la RP, que aparecen en las propuestas curriculares. Los instrumentos utilizados sacados de los libros de texto o evaluaciones anteriores son muy tradicionales y no se adecuan a los objetivos de la enseñanza actual.

Los profesores manifiestan sus dificultades para llevar al aula los cambios que se requieren que justifican en base a la formación recibida en los cursos de formación del profesorado y a las condiciones en las que desarrollan su práctica docente. A este respecto, señalan referencias concretas a los conocimientos sobre la evaluación en la RP, al desconocimiento sobre el uso de nuevos instrumentos o a la dificultad de utilizar los medios tecnológicos en el aula. Se sugiere revisar e implementar en la formación inicial y permanente de profesores de matemáticas la formación que se da sobre la resolución de problemas y los instrumentos de evaluación que se pueden emplear a este respecto.

Por ello, consideramos necesario profundizar en la relación entre las prácticas de evaluación desarrolladas por los profesores y las orientaciones actuales que señalan a la RP como contenido específico y trasversal. Estimamos que el cambio en la educación implica transformar los métodos de evaluación, de nada servirá introducir cambios curriculares si no modificamos la evaluación.

\section{Referencias}

Aksoy, Y., Bayazit, I. y Kirnap, S.M. (2015). Prospective Primary school teacherśp proficiencies in solving real-words problems: approaches, strategies and models. Eurasia Journal of Mathematics, Science \& Tecnology Education, 11(4), 827-839.

Álvarez, R. y Blanco, L.J. (2015). Evaluación en Matemáticas: Introducción al Álgebra y Ecuaciones en $1^{\circ}$ ESO. Revista Unión 42, 133-149. file:///C:/Users/Lorenzo-PC/Documents $/ 2015 \% 20 \%$ C3\%81lvarez\%20y\%20B|\%C3\%B1anco.pdf

Ayllón, M.F. (2012). Invensión-Resolución de problemas por alumnos de educación primaria. Tesis doctoral inédita. Universidad de Granada. Granada, España.

Blanco, L.J., Guerrero, E., y Caballero, A. (2013). Cognition and Affect in Mathematics Problem Solving with Prospective Teachers. The Mathematics Enthusiast. 10(1 y 2). 335-364. http://www.math.umt.edu/tmme/vol10no1and2/13-Blanco- et\%20 al_pp335_364.pdf

Cárdenas, J.A. (2014). La evaluación de la resolución de problemas en matemáticas: concepciones y prácticas de los profesores de secundaria. Tesis doctoral inédita. Universidad de Extremadura. Badajoz, España.

Cárdenas, J.A. y Blanco, L.J. (2015). La Resolución de Problemas de Matemáticas como 
La evaluación de la Resolución de Problemas de Matemáticas de profesores de Secundaria en Colombia

Janeth A. Cárdenas Lizarazo y Lorenzo J. Blanco Nieto

contenido en el Currículo de Primaria. En L.J. Blanco et al. (Eds), La resolución de problemas de Matemáticas en la Formación Inicial de profesores de primaria, (pp. 23-37). Serv. Publ. UEx.

Cárdenas, J.A., Blanco, L.J., Guerrero, E. y Gómez, R. (2013). Resolución de problemas de matemáticas y evaluación: aspectos afectivos y cognitivos. En L.J. Blanco, V. MeIlado, B. Borrachero y J.A. Cárdenas: Las emociones en la enseñanza de las matemáticas, (pp. 67-88). Badajoz: Indugrafic.

Cárdenas, J.A., Blanco, L.J., Guerrero, E. y Caballero, A. (2016). Manifestaciones de los profesores de matemáticas sobre sus prácticas de evaluación de la resolución de problemas. Revista Bolema, 30(55), 649-669.

Cárdenas, J.A., Blanco. L.J. y Cáceres, M.J. (2016). La evaluación de las matemáticas: análisis de las pruebas escritas que se realizan en secundaria. Revista UNION, 48, 59-78.

Carrillo, J. (1995). La resolución de problemas en Matemáticas: ¿cómo abordar su evaluación? Investigación en la Escuela, 25, 79-86.

Díaz, M.V. y Poblete, A. (2001). Contextualizando tipos de problemas matemátics en el aula. Números 45, 33-41.

Fortuny, J.M. (2000). Propuestas de seguimiento en educación matemática. Propuestas metodológicas y de evaluación en la formación inicial de los profesores. En C. Corral y E. Zubieta, IV simposio sobre propuestas metodológicas y de evaluación en la formación inicial de profesores de Matemáticas. Universidad de Oviedo, Oviedo.

Gil, J. (1992-93). La metodología de investigación mediante grupos de discusión. Enseñanza, $(X-X I), 199-212$.

Goñi, J. (2008). La evaluación de las competencias determinará el currículo de matemáticas. En J. Goñi, 32-2 ideas clave, (pp.167-185). El desarrollo de la competencia matemática. España: GRAO.

González, D.A., Arévalo, M., y Henao, M.V. (2009). Las evaluaciones en el aula. El caso de Colombia. Documento de circulación restringida elaborado por el Grupo de Investigación GIECE. Universidad de San Buenaventura: Cali.

Grupo de Investigación en Evaluación (2008). Informe de avance.

Harlen, W. (2012). The role of assessment in developing motivation for learning. En J. Gardner (Ed.), Assessment and Learning (pp. 171-183). California: Sage.

Jarero, M., Aparicio, E., y Sosa, L. (2013). Pruebas escritas como estrategia de evaluación de aprendizajes matemáticos. Un Estudio de caso a nivel superior. Revista Latinoamericana de Investigación en Matemática Educativa, 16(2), 213-243

Lester, F.K. (2013). Thoughts about research on mathematical problem-solving instruction. The Mathematics Enthusiast, 10(1y2), 245-278.

Lochhead, J., y Mestre, J. (1988). The language of algebra. En Yearbook of the National Council of Teachers of Mathematics. Reston, VA: National Council of Teachers of Mathematics.

Meier, S.L. (1992). Evaluating problem-solving processes. Mathematics Teacher, 85(8), 664-666.

Men (2007). Estándares Curriculares en Matemáticas. Ministerio de Educación Nacional, Bogotá, Colombia. 
La evaluación de la Resolución de Problemas de Matemáticas de profesores de Secundaria en Colombia

Janeth A. Cárdenas Lizarazo y Lorenzo J. Blanco Nieto

Mollà, A. (1997). Una experiencia de formación del profesorado en el área de matemáticas. Revista UNO, 11, 79-90.

Nikerson, R., Perkins, D., y Smith, E. (1994). Enseñar a pensar. Aspectos de la aptitud intelectual. Bacelona, Paidós.

Ortega, T., Pecharroman, C. y Sosa, P. (2011). La importancia de los enunciados de problemas matemáticos. Revista Educatio Siglo XXI, 29(2), 99-116.

Pino, J.A. (2013). La resolución de problemas y el dominio afectivo: un estudio con profesores de matemáticas de secundaria. En V. Mellado, L.J. Blanco, A.B. Borrachero y J.A. Cárdenas (Eds), Las Emociones en la Enseñanza y el Aprendizaje de las Ciencias Experimentales y de las Matemáticas (pp. 117-148). Badajoz, España: Deprofe.

Pino, J.A. y Blanco, L.J. (2008). Análisis de los problemas de los libros de texto de Matemáticas para alumnos de 12 a 14 años de edad de España y de Chile en relación con los contenidos de proporcionalidad. Publicaciones 38. 63-88.

Prieto, M., y Contreras, G. (2008). Las concepciones que orientan las prácticas evaluativas de los profesores: Un problema a develar. Estudios Pedagógicos, XXXIV(2), 245-262.

Puig, L. (1996). Elementos de resolución de problemas. España, Granada: Comares.

Puig, L. (2008) Resolución de problemas: 30 años después. En R. Luengo, B. Gomez, M. Camacho y L.J. Blanco, Investigación en Educación Matemática XII (pp. 93-111). Badajoz, España: SEIEM.

Remesal, A. (2006). La resolución de problemas en las prácticas de evaluación del área de matemáticas en la educación obligatoria: ideas de profesores y alumnos. Tesis doctoral inédita. Universidad de Barcelona. Barcelona: España.

Santos, L.M. (2007). La Resolución de Problemas matemáticos. Fundamentos cognitivos. México. Trillas.

Santos, L.M. (2012). El papel de la Resolución de problemas en el Desarrollo del Conocimiento Matemático de los Profesores para la Enseñanza. Revista Cuadernos de Investigación y Formación en Educación Matemática, 7(10), 151-163. https://revistas. ucr.ac.cr/index.php/cifem/article/viewFile/10566/10003

Sánchez-Barbero, B. (2017). Análisis de la interacción profesor alumnos al resolver problemas no rutinarios en aulas de primaria. Tesis doctoral inédita. Universidad de Salamanca.

Schoenfeld, A. (1985). Mathematical Problem Solving. Florida USA: Academic Press. Inc.

Vallejo, M. y Molina, J. (2014) La evaluación auténtica de los procesos educativos. Revista Ibero-Americana de Educação, 64, 11-25.

Woods, P. (1987). La escuela por dentro. La etnografía en la investigación educativa. Paidós-MEC. Barcelona.

Worth, J. (1990). Developing problem-solving abilities and attitudes. En Mathematics for young children (pp. 39-61). N.C.T.M. Association Drive, Reston Virginia. 
La evaluación de la Resolución de Problemas de Matemáticas de profesores de Secundaria en Colombia

Janeth A. Cárdenas Lizarazo y Lorenzo J. Blanco Nieto

\section{Anexo 1}

\begin{tabular}{|c|c|}
\hline ÍTEM & ENUNCIADO DEL ÍTEM \\
\hline 1 & $\begin{array}{l}\text { ¿Evalúo el distinguir y separar las partes de un problema hasta com- } \\
\text { prenderlo? }\end{array}$ \\
\hline 2 & $\begin{array}{l}\text { ¿Evalúo el explicar lo que personalmente se concibe o entiende del } \\
\text { problema? }\end{array}$ \\
\hline 3 & $\begin{array}{l}\text { ¿Evalúo el visualizar las relaciones existentes entre los diferentes ele- } \\
\text { mentos del problema? }\end{array}$ \\
\hline 4 & $\begin{array}{l}\text { ¿Evalúo el hacer pronósticos sobre la solución del problema plantea- } \\
\text { do? }\end{array}$ \\
\hline 5 & $\begin{array}{l}\text { ¿Evalúo el hacer uso de diversos lenguajes para expresar ideas ma- } \\
\text { temáticas pertinentes a partir de situaciones a partir de situaciones } \\
\text { problema? }\end{array}$ \\
\hline 6 & $\begin{array}{l}\text { ¿Evalúo el verbalizar los procedimientos seguidos en la resolución de } \\
\text { problemas? }\end{array}$ \\
\hline 7 & $\begin{array}{l}\text { ¿Evalúo el construir modelos gráficos o simbólicos para describir las } \\
\text { relaciones existentes entre los datos del problema? }\end{array}$ \\
\hline 8 & $\begin{array}{l}\text { ¿Evalúo el proponer más de una estrategia a seguir de acuerdo con las } \\
\text { condiciones del problema? }\end{array}$ \\
\hline 9 & $\begin{array}{l}\text { ¿Evalúo el seleccionar recursos materiales apropiados para resolver } \\
\text { problemas? }\end{array}$ \\
\hline 10 & $\begin{array}{l}\text { ¿Evalúo el estructurar argumentos sobre las acciones desarrolladas en } \\
\text { la resolución de problemas? }\end{array}$ \\
\hline 11 & ¿Evalúo el validar el modelo planteado para la solución del problema? \\
\hline 12 & $\begin{array}{l}\text { ¿Evalúo el implementar recursos apropiados en la resolución de pro- } \\
\text { blemas? }\end{array}$ \\
\hline 13 & ¿Evalúo el hacer cálculos de rutina para la solución de problemas? \\
\hline 14 & $\begin{array}{l}\text { ¿Evalúo el aplicar métodos matemáticos trabajados en clase en la } \\
\text { resolución de problemas? }\end{array}$ \\
\hline 15 & $\begin{array}{l}\text { ¿Evalúo el usar estrategias o métodos diferentes a los que se han traba- } \\
\text { jado en clase para la resolución de problemas? }\end{array}$ \\
\hline 16 & $\begin{array}{l}\text { ¿Evalúo el validar los pronósticos formulados acerca de la solución } \\
\text { del problema? }\end{array}$ \\
\hline 17 & $\begin{array}{l}\text { ¿Evalúo el ajustar o modificar el plan implementado para la resolución } \\
\text { del problema en caso de ser necesario? }\end{array}$ \\
\hline 18 & $\begin{array}{l}\text { ¿Evalúo el reconocer si la respuesta que requiere un problema es un } \\
\text { dato exacto o un dato aproximado? }\end{array}$ \\
\hline 19 & $\begin{array}{l}\text { ¿Evalúo el trasladar a la situación problema los resultados, para ser } \\
\text { interpretados en relación con ella? }\end{array}$ \\
\hline 20 & $\begin{array}{l}\text { ¿Evalúo el validar los resultados a través del proceso matemático } \\
\text { empleado? }\end{array}$ \\
\hline
\end{tabular}


La evaluación de la Resolución de Problemas de Matemáticas de profesores de Secundaria en Colombia

Janeth A. Cárdenas Lizarazo y Lorenzo J. Blanco Nieto

\begin{tabular}{|c|c|}
\hline ÍTEM & ENUNCIADO DEL ÍTEM \\
\hline 21 & $\begin{array}{l}\text { ¿Evalúo la visión que los estudiantes tienen de las matemáticas en la } \\
\text { resolución de problemas? }\end{array}$ \\
\hline 22 & $\begin{array}{l}\text { ¿Evalúo las actitudes que se presentan ante el estímulo de tareas que } \\
\text { pueden ofrecer un carácter problemático? }\end{array}$ \\
\hline 23 & $\begin{array}{l}\text { ¿Evalúo la visión que cada estudiante tiene de sí mismo en la resolu- } \\
\text { ción de problemas? }\end{array}$ \\
\hline 24 & $\begin{array}{l}\text { ¿Evalúo el desarrollo de la confianza en sí mismo para hacer frente a } \\
\text { situaciones problema? }\end{array}$ \\
\hline 25 & $\begin{array}{l}\text { ¿Evalúo el realizar la resolución de problemas de manera indepen- } \\
\text { diente? }\end{array}$ \\
\hline 26 & $\begin{array}{l}\text { ¿Evalúo la confianza en el uso de las matemáticas al matematizar un } \\
\text { problema? }\end{array}$ \\
\hline 27 & $\begin{array}{l}\text { ¿Evalúo las técnicas o estrategias que usa o evita en la resolución de } \\
\text { problemas? }\end{array}$ \\
\hline 28 & ¿Evalúo el esfuerzo que dedica en la resolución de problemas? \\
\hline 29 & $\begin{array}{l}\text { ¿Evalúo el extraer conclusiones de los procesos seguidos en la resolu- } \\
\text { ción del problema? }\end{array}$ \\
\hline 30 & $\begin{array}{l}\text { ¿En la evaluación de la resolución de problemas propongo problemas } \\
\text { con diferentes contextos Matemáticos? }\end{array}$ \\
\hline 31 & $\begin{array}{l}\text { ¿En la evaluación de la resolución de problemas propongo problemas } \\
\text { con contextos no Matemáticos? }\end{array}$ \\
\hline 32 & $\begin{array}{l}\text { ¿En la evaluación de la resolución de problemas propongo problemas } \\
\text { sin solución alguna? }\end{array}$ \\
\hline 33 & $\begin{array}{l}\text { ¿En la evaluación de la resolución de problemas propongo problemas } \\
\text { con una única solución? }\end{array}$ \\
\hline 34 & $\begin{array}{l}\text { ¿En la evaluación de la resolución de problemas propongo problemas } \\
\text { con más de una solución pero seleccionando o buscando solo una? }\end{array}$ \\
\hline 35 & $\begin{array}{l}\text { ¿En la evaluación de la resolución de problemas propongo problemas } \\
\text { con más de una solución, encontrando más de una solución? }\end{array}$ \\
\hline 36 & $\begin{array}{l}\text { ¿En la evaluación de la resolución de problemas propongo problemas } \\
\text { con más de una solución, encontrando todas las soluciones posibles? }\end{array}$ \\
\hline 37 & $\begin{array}{l}\text { ¿Para conocer cómo van los procesos de resolución de problemas de } \\
\text { los estudiantes, tengo en cuenta lo que ellos comprenden del proble- } \\
\text { ma? }\end{array}$ \\
\hline 38 & $\begin{array}{l}\text { ¿Para conocer cómo van los procesos de resolución de problemas de } \\
\text { los estudiantes, tengo en cuenta lo que ellos saben de resolución de } \\
\text { problemas? }\end{array}$ \\
\hline 39 & $\begin{array}{l}\text { ¿Para conocer cómo van los procesos de resolución de problemas de } \\
\text { los estudiantes, tengo en cuenta lo que ellos piensan sobre la resolu- } \\
\text { ción de problemas? }\end{array}$ \\
\hline
\end{tabular}


La evaluación de la Resolución de Problemas de Matemáticas de profesores de Secundaria en Colombia

Janeth A. Cárdenas Lizarazo y Lorenzo J. Blanco Nieto

\begin{tabular}{|c|c|}
\hline ÍTEM & ENUNCIADO DEL ÍTEM \\
\hline 40 & $\begin{array}{l}\text { ¿Para conocer cómo van los procesos de resolución de problemas de } \\
\text { los estudiantes, tengo en cuenta lo que ellos piensan de su capacidad } \\
\text { de resolver problemas? }\end{array}$ \\
\hline 41 & $\begin{array}{l}\text { ¿Para conocer cómo van los procesos de resolución de problemas de } \\
\text { los estudiantes, tengo en cuenta las preguntas que hacen para resolver } \\
\text { problemas? }\end{array}$ \\
\hline 42 & $\begin{array}{l}\text { ¿Al momento de plantear la evaluación de la resolución de problemas } \\
\text { tengo presente el nivel de los conocimientos matemáticos con los que } \\
\text { cuentan los estudiantes es accesible al tratamiento matemático que } \\
\text { requiere el problema planteado? }\end{array}$ \\
\hline 43 & $\begin{array}{l}\text { ¿Al momento de plantear la evaluación de la resolución de proble- } \\
\text { mas tengo presente que las situaciones problemáticas diseñadas sean } \\
\text { coherentes con los logros de aprendizaje propuestos en el diseño } \\
\text { curricular de la institución? }\end{array}$ \\
\hline 44 & $\begin{array}{l}\text { ¿En la evaluación de la resolución de problemas propongo a mis } \\
\text { alumnos Inventar o diseñar problemas? }\end{array}$ \\
\hline 45 & $\begin{array}{l}\text { ¿En la evaluación de la resolución de problemas propongo a mis } \\
\text { alumnos formular problemas a partir de una situación dada? }\end{array}$ \\
\hline 46 & $\begin{array}{l}\text { ¿En la evaluación de la resolución de problemas propongo a mis } \\
\text { alumnos reformular o plantear el mismo problema en otras palabras? }\end{array}$ \\
\hline 47 & $\begin{array}{l}\text { ¿En la evaluación de la resolución de problemas propongo a mis } \\
\text { alumnos transformar o cambiar el problema a partir del problema } \\
\text { dado? }\end{array}$ \\
\hline 48 & $\begin{array}{l}\text { ¿Entre las dificultades que muestran los estudiantes para plantear o } \\
\text { resolver un problema cuando evalúo, determino si son dificultades en } \\
\text { la comprensión del problema? }\end{array}$ \\
\hline 49 & $\begin{array}{l}\text { ¿Entre las dificultades que muestran los estudiantes para plantear o } \\
\text { resolver un problema cuando evalúo, determino si son dificultades en } \\
\text { el conocimiento matemático? }\end{array}$ \\
\hline 50 & $\begin{array}{l}\text { ¿Entre las dificultades que muestran los estudiantes para plantear o } \\
\text { resolver un problema cuando evalúo, determino si son dificultades en } \\
\text { los procesos para aplicar algoritmos? }\end{array}$ \\
\hline 51 & $\begin{array}{l}\text { ¿Entre las dificultades que muestran los estudiantes para plantear o } \\
\text { resolver un problema cuando evalúo, determino si son dificultades en } \\
\text { las actitudes sociales? }\end{array}$ \\
\hline 52 & $\begin{array}{l}\text { ¿Entre las dificultades que muestran los estudiantes para plantear o } \\
\text { resolver un problema cuando evalúo, determino si son dificultades } \\
\text { culturales? }\end{array}$ \\
\hline 53 & $\begin{array}{l}\text { ¿Entre las dificultades que muestran los estudiantes para plantear o } \\
\text { resolver un problema cuando evalúo, determino si son dificultades } \\
\text { emocionales? }\end{array}$ \\
\hline
\end{tabular}


La evaluación de la Resolución de Problemas de Matemáticas de profesores de Secundaria en Colombia

Janeth A. Cárdenas Lizarazo y Lorenzo J. Blanco Nieto

\begin{tabular}{|c|c|}
\hline ÍTEM & ENUNCIADO DEL ÍTEM \\
\hline 54 & $\begin{array}{l}\text { ¿Entre las dificultades que muestran los estudiantes para plantear o } \\
\text { resolver un problema cuando evalúo, determino si son dificultades } \\
\text { hacia la matemática? }\end{array}$ \\
\hline 55 & $\begin{array}{l}\text { ¿Entre las dificultades que muestran los estudiantes para plantear o } \\
\text { resolver un problema cuando evalúo, determino si son dificultades } \\
\text { hacia la resolución de problemas? }\end{array}$ \\
\hline 56 & $\begin{array}{l}\text { ¿Entre las dificultades que muestran los estudiantes para plantear o } \\
\text { resolver un problema cuando evalúo, determino si son dificultades } \\
\text { hacia el modificar los problemas en términos matemáticos? }\end{array}$ \\
\hline 57 & $\begin{array}{l}\text { ¿Entre las dificultades que muestran los estudiantes para plantear o } \\
\text { resolver un problema cuando evalúo, determino si se tiene tendencia } \\
\text { a dar respuestas estereotipadas (responder con el esquema disponible, } \\
\text { aunque el problema no esté relacionado con el esquema)? }\end{array}$ \\
\hline 58 & $\begin{array}{l}\text { Doy importancia a que mis estudiantes al resolver problemas obten- } \\
\text { gan el resultado exacto }\end{array}$ \\
\hline 59 & $\begin{array}{l}\text { Doy importancia a que mis estudiantes al resolver problemas utilicen } \\
\text { las cosas que acabamos de explicar }\end{array}$ \\
\hline 60 & $\begin{array}{l}\text { Doy importancia a que mis estudiantes al resolver problemas expli- } \\
\text { quen por qué hacen cada cosa }\end{array}$ \\
\hline 61 & $\begin{array}{l}\text { Doy importancia a que mis estudiantes al resolver problemas sigan el } \\
\text { camino que yo quiero }\end{array}$ \\
\hline 62 & $\begin{array}{l}\text { Doy importancia a que mis estudiantes al resolver problemas al aca- } \\
\text { bar, ver si había otros caminos }\end{array}$ \\
\hline 63 & $\begin{array}{l}\text { Cuando miro cómo resuelve un alumno un problema, doy importan- } \\
\text { cia a que desde el principio vaya por el buen camino }\end{array}$ \\
\hline 64 & $\begin{array}{l}\text { Cuando miro cómo resuelve un alumno un problema, doy importan- } \\
\text { cia a que lo resuelva en la cabeza antes de escribir nada }\end{array}$ \\
\hline 65 & $\begin{array}{l}\text { Cuando miro cómo resuelve un alumno un problema, doy importan- } \\
\text { cia a que no se quede bloqueado en ningún momento }\end{array}$ \\
\hline 66 & $\begin{array}{l}\text { Cuando miro cómo resuelve un alumno un problema, doy importan- } \\
\text { cia a que lo haya resuelto en poco tiempo }\end{array}$ \\
\hline 67 & $\begin{array}{l}\text { Cuando planteo una cuestión en clase de matemáticas estoy esperan- } \\
\text { do que alguien recuerde la respuesta correcta y responda }\end{array}$ \\
\hline 68 & $\begin{array}{l}\text { Cuando planteo una cuestión en clase de matemáticas estoy esperan- } \\
\text { do que discutan mis estudiantes antes de intentar dar una respuesta }\end{array}$ \\
\hline 69 & $\begin{array}{l}\text { Cuando planteo una cuestión en clase de matemáticas estoy esperan- } \\
\text { do ver quiénes son los que han estudiado y trabajado }\end{array}$ \\
\hline 70 & $\begin{array}{l}\text { Cuando planteo una cuestión en clase de matemáticas estoy espe- } \\
\text { rando que mis estudiantes piensen en torno a ello. No siempre estoy } \\
\text { esperando una respuesta }\end{array}$ \\
\hline
\end{tabular}


La evaluación de la Resolución de Problemas de Matemáticas de profesores de Secundaria en Colombia

Janeth A. Cárdenas Lizarazo y Lorenzo J. Blanco Nieto

\begin{tabular}{cl}
\hline ITTEM & \multicolumn{1}{c}{ ENUNCIADO DEL ÍTEM } \\
\hline $\mathbf{7 1}$ & $\begin{array}{l}\text { Para enseñar a resolver problemas me da la sensación que tengo que } \\
\text { enseñar muchas matemáticas }\end{array}$ \\
\hline $\mathbf{7 2}$ & $\begin{array}{l}\text { Para enseñar a resolver problemas me da la sensación que tengo que } \\
\text { enseñar a ser intuitivo y a utilizar el sentido común }\end{array}$ \\
\hline $\mathbf{7 3}$ & $\begin{array}{l}\text { Para enseñar a resolver problemas me da la sensación que tengo que } \\
\text { enseñar a dominar el estado de ánimo de cada alumno }\end{array}$ \\
\hline $\mathbf{7 4}$ & $\begin{array}{l}\text { Para enseñar a resolver problemas me da la sensación que tengo que } \\
\text { enseñar estrategias como por ejemplo hacer esquemas, representacio- } \\
\text { nes,... }\end{array}$ \\
\hline $\mathbf{7 5}$ & $\begin{array}{l}\text { Para enseñar a resolver problemas me da la sensación que tengo que } \\
\text { enseñar estrategias como por ejemplo probar con casos más sencillos, } \\
\text { con ejemplos... }\end{array}$ \\
\hline $\mathbf{7 6}$ & $\begin{array}{l}\text { Considero que es más importante resolver problemas en los que el } \\
\text { enunciado no tiene pistas sobre qué hace falta hacer para resolverlo }\end{array}$ \\
\hline $\mathbf{7 7}$ & $\begin{array}{l}\text { Considero que es más importante resolver problemas en los que el } \\
\text { enunciado no tiene ninguna palabra }\end{array}$ \\
\hline $\mathbf{7 8}$ & $\begin{array}{l}\text { Considero que es más importante resolver problemas en los que al } \\
\text { enunciado le faltan datos que se necesitan para poder resolver el } \\
\text { problema }\end{array}$ \\
\hline $\mathbf{7 9}$ & $\begin{array}{l}\text { Considero que es más importante resolver problemas en los que el } \\
\text { enunciado tiene datos que no se necesitan para nada }\end{array}$ \\
\hline $\mathbf{8 0}$ & $\begin{array}{l}\text { Considero que es más importante resolver problemas en los que el } \\
\text { enunciado es poco claro }\end{array}$ \\
\hline $\mathbf{8 1}$ & $\begin{array}{l}\text { En los enunciados de problemas de matemáticas que normalmente } \\
\text { planteo para trabajar en clase doy expresiones y nombres de cosas } \\
\text { matemáticas }\end{array}$ \\
\hline $\mathbf{8 2}$ & $\begin{array}{l}\text { En los enunciados de problemas de matemáticas que normalmente } \\
\text { planteo para trabajar en clase doy pistas sobre lo que se debe hacer } \\
\text { para resolverlos }\end{array}$ \\
\hline $\mathbf{8 4}$ & $\begin{array}{l}\text { En los enunciados de problemas de matemáticas que normalmente } \\
\text { planteo para trabajar en clase doy todos los datos que se necesitan } \\
\text { para resolver el problema }\end{array}$ \\
\hline $\begin{array}{l}\text { En los enunciados de problemas de matemáticas que normalmente } \\
\text { planteo para trabajar en clase doy datos que no se necesitan }\end{array}$ \\
\hline $\begin{array}{l}\text { En los enunciados de problemas de matemáticas que normalmente } \\
\text { planteo para trabajar en clase doy datos y condiciones muy claros y } \\
\text { exactos }\end{array}$ \\
\hline
\end{tabular}

